\title{
Direct Beamformer Estimation for Dynamic TDD Networks with Forward-Backward Training
}

\author{
Praneeth Jayasinghe, Antti Tölli, Jarkko Kaleva and Matti Latva-aho \\ Centre for Wireless Communications, University of Oulu, P.O. Box 4500, FIN-90014, Oulu, Finland. \\ \{praneeth.laddu, antti.tolli, jarkko.kaleva, matti.latva-aho\}@oulu.fi
}

\begin{abstract}
This paper investigates direct beamformer estimation in dynamic time division duplexing (TDD) system with the objective of weighted sum rate maximization. For a given TDD frame, base stations (BS) are allocated to either uplink or downlink based on the instantaneous traffic state. The weighted sum mean-squared error minimization framework is used to obtain the decentralized iterative solution for the beamformer optimization. The received precoded pilot training matrices are directly used in the decoupled optimization problem, and two different over the air bi-directional signaling strategies are used for iterative forward-backward training of both transmit and receive beamformers. Detailed flow of signaling exchange and the beamformers estimation procedures are described for each bi-directional signaling strategy. Moreover, the proposed beamformer signaling schemes allow non-orthogonal and overlapping pilots, which greatly reduces the resource allocation effort. The numerical examples illustrate the system performance of the proposed algorithms against the training sequence length.
\end{abstract}

\section{INTRODUCTION}

With the ever growing mobile traffic, future mobile networks should be able to satisfy asymmetric and bursty traffic generated in mobile data networks. This can be accomplished with the use of a large number of small cells with very short coverage in addition to macro/micro cells, in high mobile data traffic areas [1]. Furthermore, time division duplexing (TDD) systems can be employed to provide unbalanced mobile data traffic. Therefore, small-cell networks are more suited with TDD mode to obtain various benefits for network designing, such as reduced complexity of estimating the channel state information (CSI), flexible dynamic traffic handling, and easy frequency planning [2].

Dynamic TDD can be used to satisfy unbalanced instantaneous mobile data traffic by adjusting the uplink (UL)/downlink (DL) mode asynchronously over the network based on the traffic generated within each cell [3]. Consequently, the dynamic TDD system vastly improves the overall resource utilization of the small-cell network when compared to the network with traditional fixed UL/DL time slot allocation across every cell [4]. Fully dynamic or flexible TDD is an essential $5 \mathrm{G}$ research item. So far there has been good progress in 3GPP new radio (NR) study item [5] in this direction. However, complicated cases arise with dynamic

This research was supported by the Finnish Funding Agency for Technology and Innovation (TEKES), Nokia and Infotech Oulu Graduate School.
TDD systems, such as the DL BS transmission is interfering the UL BS reception (BS-BS interference) and the UL user transmission is interfering the DL user reception (user-user interference) [6]. Thus, the interference management becomes more challenging.

Most of the dynamic TDD studies were focused on proposing complicated time slot allocation algorithms to mitigate this cross-link interference associated with dynamic TDD system [7]-[9]. Multiple-input multiple-output (MIMO) systems can be used to mitigate the interference induced in dynamic TDD systems with the coordinated beamformer design. In the context of static TDD mode, numerous types of beamformer designs were proposed to optimize the network utilities such as weighted sum rate (WSR), energy efficiency and the total number of backlogged packets in multi-cell multi-user MIMO systems [10]-[13]. Centralized and decentralized beamformer design has been considered with different coordination assumptions. While the channel reciprocity can be utilized to acquire the CSI of the user-BS and BS-BS links, a specific challenge of the dynamic TDD approach is to acquire the CSI between the mutually interfering user terminals which renders the centralized design impractical.

In [14], [15], we introduced different bi-directional over-theair (OTA) signaling strategies to facilitate iterative beamformer design with the objective to maximize the WSR of multicell multi-user MIMO dynamic TDD system. Bi-directional signaling was embedded into TDD frame to exchange equivalent channel information between coordinated nodes. Each node uses the independently optimized beamformers as pilot precoders for the next forward/backward iteration. However, perfect estimation of CSI at the BSs/users was assumed in those beamformer design algorithms. Nevertheless, in a dense dynamic TDD network, the number of orthogonal pilots that can be used for channel estimation is limited due to coherence time and coherence bandwidth of the wireless channel [16]. In [17], direct least squares (LS) beamformer estimation from the contaminated UL/DL pilots was shown to provide good performance as opposed to the estimation of the individual channels. In this paper, we further investigate proposed bidirectional strategies to maximize WSR in dynamic TDD network in [14], [15] for imperfect pilot conditions by introducing pilot aided direct estimation (DE) methods similarly to [17]. 
The resource allocation is performed independently at each base station (BS) and the TDD frames are assumed to be either in UL or DL mode depending on the instantaneous traffic demand. Moreover, pilot training sequences used in each node are not necessarily known to others. Hence, we modify the original decoupled optimization problem to solve beamformers directly from the received composite pilot response matrix at each node. A numerical study is carried out to investigate the performance of each bi-directional signaling strategy under worst circumstances by using non-orthogonal and overlapping pilot sequences.

\section{SySTEM MODEL}

We consider a multi-cell multi-user MIMO system operating in dynamic TDD mode. The multi-cell network consists of $N$ BSs and $K$ users. We denote the set of BS indices as $\mathcal{B}$ while the set of users served by BS $i$ denoted $\mathcal{U}_{i}$. Each user $k$ employs $N_{k}$ antenna elements, whereas each BS $i$ employs $M_{i}$ antenna elements. In a given time, a subset of base stations $\mathcal{B}_{U} \subseteq \mathcal{B}$ serves the uplink traffic and rest of the base stations $\mathcal{B}_{D} \subseteq \mathcal{B}$ are serving the DL traffic. The maximum number of spatial data streams allocated to user $k \in \mathcal{U}_{i}$ is denoted by $L_{k} \leq \min \left(M_{i}, N_{k}\right)$. Furthermore, we assume channel reciprocity of the wireless channel to obtain the CSI. The received signal $\mathbf{x}_{k} \in \mathbb{C}^{N_{k}}$ at the DL user $k$ can be expressed as

$$
\begin{aligned}
\mathbf{x}_{k}= & \sum_{i \in \mathcal{B}_{D}} \sum_{j \in \mathcal{U}_{i}} \sum_{l=1}^{L_{j}} \mathbf{H}_{i, k} \mathbf{m}_{i, j, l} d_{j, l}^{D} \\
& +\sum_{q \in \mathcal{B}_{U}} \sum_{r \in \mathcal{U}_{q}} \sum_{s=1}^{L_{r}} \tilde{\mathbf{H}}_{r, k} \mathbf{v}_{q, r, s} d_{r, s}^{U}+\mathbf{z}_{k},
\end{aligned}
$$

where $\mathbf{H}_{i, k} \in \mathbb{C}^{N_{k} \times M_{i}}$ is the channel matrix between the DL BS $i$ and the user $k, \tilde{\mathbf{H}}_{r, k} \in \mathbb{C}^{N_{k} \times N_{r}}$ is the interference channel matrix between the UL user $r$ and the DL user $k$. The transmit precoder for $l^{\text {th }}$ spatial data stream of the DL user $j \in \mathcal{U}_{i}$ is denoted as $\mathbf{m}_{i, j, l} \in \mathbb{C}^{M_{i}}$ and the transmit precoder for $s^{t h}$ spatial data stream of the UL user $r \in \mathcal{U}_{q}$ is $\mathbf{v}_{q, r, s} \in \mathbb{C}^{N_{s}}$. Transmitted data symbols to DL user $j$ in $l^{t h}$ spatial stream and transmitted data symbols from UL user $r$ in $s^{t h}$ spatial stream are denoted as $d_{j, l}^{D}$ and $d_{r, s}^{U}$, respectively. Here, the transmit data symbols assumed to be independent and identically distributed with $\mathbb{E}\left\{\left|d_{k, l}^{D}\right|^{2}\right\}=1$ and $\mathbb{E}\left\{\left|d_{r, s}^{U}\right|^{2}\right\}=1$. We assume complex white Gaussian noise vector $\mathbf{z}_{k} \in \mathbb{C}^{N_{k}}$ with variance $\mathcal{N}_{0}$ per element. Similarly, the received signal $\mathbf{y}_{p} \in \mathbb{C}^{M_{p}}$ at the uplink BS $p$ is given by

$$
\begin{aligned}
\mathbf{y}_{p}= & \sum_{q \in \mathcal{B}_{U}} \sum_{r \in \mathcal{U}_{q}} \sum_{s=1}^{L_{r}} \mathbf{H}_{p, r}^{\mathrm{T}} \mathbf{v}_{q, r, s} d_{r, s}^{U} \\
& +\sum_{i \in \mathcal{B}_{D}} \sum_{j \in \mathcal{U}_{i}} \sum_{l=1}^{L_{j}} \hat{\mathbf{H}}_{i, p} \mathbf{m}_{i, j, l} d_{j, l}^{D}+\mathbf{z}_{p},
\end{aligned}
$$

where $\hat{\mathbf{H}}_{i, p} \in \mathbb{C}^{M_{p} \times M_{i}}$ is the interference channel matrix between the DL BS $i$ and the UL BS $p$. Also, we consider $\mathbf{z}_{p} \in \mathbb{C}^{M_{p}}$ is a complex white Gaussian noise vector with variance $\mathcal{N}_{0}$ per element.
At the DL user $k$, we employ a linear receiver $\mathbf{u}_{k, l} \in \mathbb{C}^{N_{k}}$. The estimated data becomes $\hat{d}_{k, l}^{D}=\mathbf{u}_{k, l}^{\mathrm{H}} \mathbf{x}_{k}$. Then, the meansquared error (MSE) $\epsilon_{k, l}^{\mathrm{D}}=\mathbb{E}\left[\left|d_{k, l}^{D}-\hat{d}_{k, l}^{D}\right|^{2}\right]$ for the spatial data stream $l$ of the DL user $k$ is given by

$$
\epsilon_{k, l}^{D}=1-2 \Re\left(\mathbf{u}_{k, l}^{\mathrm{H}} \mathbf{H}_{i, k} \mathbf{m}_{i, k, l}\right)+\mathbf{u}_{k, l}^{\mathrm{H}} \mathbf{M}_{k}^{D} \mathbf{u}_{k, l},
$$

where $\mathbf{M}_{k}^{D}=\mathbb{E}\left[\mathbf{x}_{k} \mathbf{x}_{k}^{\mathrm{H}}\right]$ is the received signal covariance matrix at the DL user $k$. Which is given by

$$
\begin{aligned}
\mathbf{M}_{k}^{D}= & \sum_{i \in \mathcal{B}_{D}} \sum_{j \in \mathcal{U}_{i}} \sum_{l=1}^{L_{j}} \mathbf{H}_{i, k} \mathbf{m}_{i, j, l}\left(\mathbf{H}_{i, k} \mathbf{m}_{i, j, l}\right)^{\mathrm{H}} \\
& +\sum_{q \in \mathcal{B}_{U}} \sum_{r \in \mathcal{U}_{q}} \sum_{s=1}^{L_{r}} \tilde{\mathbf{H}}_{r, k} \mathbf{v}_{q, r, s}\left(\tilde{\mathbf{H}}_{r, k} \mathbf{v}_{q, r, s}\right)^{\mathrm{H}}+\mathcal{N}_{0} \mathbf{I} .
\end{aligned}
$$

The linear minimum MSE (MMSE) receiver for the DL user $k$ can be obtained from (3) as

$$
\mathbf{u}_{k, l}^{\mathrm{MMSE}}=\left(\mathbf{M}_{k}^{D}\right)^{-1} \mathbf{H}_{i, k} \mathbf{m}_{i, k, l} .
$$

The MSE $\tilde{\epsilon}_{k, l}^{D}$ when the MMSE receiver is employed, can be obtain as

$$
\tilde{\epsilon}_{k, l}^{D}=1-\left(\mathbf{u}_{k, l}^{\mathrm{MMSE}}\right)^{\mathrm{H}} \mathbf{H}_{i, k} \mathbf{m}_{i, k, l}
$$

which is called the MMSE value of the DL user $k$ from spatial stream $l$. Similarly, the UL BS $p$ employs a linear combining vector $\mathbf{w}_{r, s} \in \mathbb{C}^{M_{p}}$ to decode the received data from the UL user $r \in \mathcal{U}_{p}$ through the spatial stream $s$. The corresponding MSE $\epsilon_{r, s}^{\mathrm{U}}=\mathbb{E}\left[\left|d_{r, s}^{U}-\hat{d}_{r, s}^{U}\right|^{2}\right]$ is given by

$$
\epsilon_{r, s}^{U}=1-2 \Re\left(\mathbf{w}_{r, s}^{\mathrm{H}} \mathbf{H}_{p, r}^{\mathrm{T}} \mathbf{v}_{p, r, s}\right)+\mathbf{w}_{r, s}^{\mathrm{H}} \mathbf{M}_{p}^{U} \mathbf{w}_{r, s},
$$

where the received signal covariance matrix $\mathbf{M}_{p}^{U}=\mathbb{E}\left[\mathbf{y}_{p} \mathbf{y}_{p}^{\mathrm{H}}\right]$ is given by,

$$
\begin{aligned}
\mathbf{M}_{p}^{U}= & \sum_{q \in \mathcal{B}_{U}} \sum_{r \in \mathcal{U}_{q}} \sum_{s=1}^{L_{r}} \mathbf{H}_{p, r}^{\mathrm{T}} \mathbf{v}_{q, r, s}\left(\mathbf{H}_{p, r}^{\mathrm{T}} \mathbf{v}_{q, r, s}\right)^{\mathrm{H}} \\
& +\sum_{i \in \mathcal{B}_{D}} \sum_{j \in \mathcal{U}_{i}} \sum_{l=1}^{L_{j}} \hat{\mathbf{H}}_{i, p} \mathbf{m}_{i, j, l}\left(\hat{\mathbf{H}}_{i, p} \mathbf{m}_{i, j, l}\right)^{\mathrm{H}}+\mathcal{N}_{0} \mathbf{I} .
\end{aligned}
$$

The MMSE receiver and the corresponding MMSE for the UL user transmission are

$$
\begin{gathered}
\mathbf{w}_{r, s}^{\mathrm{MMSE}}=\left(\mathbf{M}_{p}^{U}\right)^{-1} \mathbf{H}_{p, r}^{\mathrm{T}} \mathbf{v}_{p, r, s}, \\
\tilde{\epsilon}_{r, s}^{U}=1-\left(\mathbf{w}_{r, s}^{\mathrm{MMSE}}\right)^{\mathrm{H}} \mathbf{H}_{p, r}^{\mathrm{T}} \mathbf{v}_{p, r, s} .
\end{gathered}
$$

\section{Problem Formulation}

We study the optimization problem to maximize the WSR of the dynamic TDD system subject to individual transmit power constraints at the DL BSs and the UL users. The derivation follows the same framework as presented in [11], [12], [14]. Assuming optimal MMSE receivers, the WSR maximization problem can be formulated equivalently with the weighted MSE framework as

$$
\begin{array}{ll}
\min _{\substack{\mathbf{m}_{i, j, l}, r \\
\mathbf{v}_{q, r}}} & \sum_{\substack{i \in \mathcal{B}_{D}, j \in \mathcal{U}_{i} \\
l \in\left\{1, \ldots, L_{j}\right\}}} \alpha_{j} \log \left(\tilde{\epsilon}_{j, l}^{D}\right)+\sum_{\substack{q \in \mathcal{B}_{U}, r \in \mathcal{U}_{q} \\
s \in\left\{1, . ., L_{q}\right\}}} \alpha_{r} \log \left(\tilde{\epsilon}_{r, s}^{U}\right) \\
\text { s. t. } & \sum_{j \in \mathcal{U}_{i}} \sum_{l=1}^{L_{j}}\left\|\mathbf{m}_{i, j, l}\right\|^{2} \leq P_{i}^{D} \quad \forall i \in \mathcal{B}_{D} \\
& \sum_{s=1}^{L_{r}}\left\|\mathbf{v}_{q, r, s}\right\|^{2} \leq P_{q, r}^{U} \quad \forall r \in \mathcal{U}_{q}, q \in \mathcal{B}_{U},
\end{array}
$$

where $\alpha_{j}, \alpha_{r}$ are the priority weights of the users. Also, $P_{i}^{D}$ and $P_{r}^{U}$ are the maximum transmit power available at the DL 
BS $i$ and at the UL user $r \in \mathcal{U}_{q}$, respectively. This WSR problem is an NP-hard problem, but a locally optimal solution can be obtained with successive convex approximation as the detailed approach described in [10]-[12], [14]. Hence, the main steps are briefly presented here for clarity. For a fixed transmit precoder $\left(\mathbf{m}_{i, j, l} / \mathbf{v}_{q, r, s}\right)$ the optimal receivers can be obtained from (5) and (9). Then, for fixed MMSE receivers and user specific linearization coefficients (MSE user weights) $\beta_{j, l}^{D}$ and $\beta_{r, s}^{U}$, transmit precoders can be obtained from the the optimization problem

$$
\begin{aligned}
& \min _{\substack{\mathbf{m}_{i, j, l} \\
\mathbf{v}_{q, r, s}}} \sum_{\substack{i \in \mathcal{B}_{D}, j \in \mathcal{U}_{i} \\
l \in\left\{1, . ., L_{j}\right\}}} \alpha_{j} \beta_{j, l}^{D} \epsilon_{j, l}^{D}+\sum_{\substack{q \in \mathcal{B}_{U}, r \in \mathcal{U}_{q} \\
s \in\left\{1, \ldots, L_{q}\right\}}} \alpha_{r} \beta_{r, s}^{U} \epsilon_{r, s}^{U} \\
& \text { s.t } \quad(11 \mathrm{~b}),(11 \mathrm{c}),
\end{aligned}
$$

where the MSE user weight of the DL data streams and UL data streams are given by

$$
\beta_{j, l}^{D}=\left(\tilde{\epsilon}_{j, l}^{D}\right)^{-1}, \beta_{r, s}^{U}=\left(\tilde{\epsilon}_{r, s}^{U}\right)^{-1} .
$$

The problem (12) is separable with respect to the optimization variables $\mathbf{m}_{i, j, l}$ and $\mathbf{v}_{q, r, s}$. Hence, the optimization problem to find the optimal DL transmit precoder $\mathbf{m}_{i, j, l}$ at the DL BS $i$ is given by

$$
\begin{array}{ll}
\min _{\mathbf{m}_{i, j, l}} & \sum_{j \in \mathcal{U}_{i}} \sum_{l=1}^{L_{j}}\left(\mathbf{m}_{i, j, l}^{\mathrm{H}} \mathbf{\Phi}_{i} \mathbf{m}_{i, j, l}-2 \Re\left(\mathbf{c}_{i, j, l} \mathbf{m}_{i, j, l}\right)\right) \\
\text { s. t. } & \sum_{j \in \mathcal{U}_{i}} \sum_{l=1}^{L_{j}}\left\|\mathbf{m}_{i, j, l}\right\|^{2} \leq P_{i}^{D},
\end{array}
$$

where $\mathbf{c}_{i, j . l}=\alpha_{j} \beta_{j, l}^{D} \mathbf{u}_{j, l}^{\mathrm{H}} \mathbf{H}_{i, j}$, and $\boldsymbol{\Phi}_{i}$ is the DL BS specific matrix. Which is given by

$$
\begin{aligned}
\boldsymbol{\Phi}_{i}= & \sum_{i \in \mathcal{B}_{D}} \sum_{j \in \mathcal{U}_{i}} \sum_{l=1}^{L_{j}} \alpha_{j} \beta_{j, l}^{D} \mathbf{H}_{i, j}^{\mathrm{H}} \mathbf{u}_{j, l}\left(\mathbf{H}_{i, j}^{\mathrm{H}} \mathbf{u}_{j, l}\right)^{\mathrm{H}} \\
& +\sum_{q \in \mathcal{B}_{U}} \sum_{r \in \mathcal{U}_{q}} \sum_{s=1}^{L_{r}} \alpha_{r} \beta_{r, s}^{U} \hat{\mathbf{H}}_{i, q}^{\mathrm{H}} \mathbf{w}_{r, s}\left(\hat{\mathbf{H}}_{i, q}^{\mathrm{H}} \mathbf{w}_{r, s}\right)^{\mathrm{H}} .
\end{aligned}
$$

The optimal solution for $\mathbf{m}_{i, j, l}$ can be obtained directly via Karush-Kuhn-Tucker (KKT) conditions as

$$
\mathbf{m}_{i, j, l}=\left(\boldsymbol{\Phi}_{i}+\lambda_{i} \mathbf{I}\right)^{-1} \mathbf{c}_{i, j, l}^{\mathrm{H}}
$$

where the optimal $\lambda_{i}$ is found by bisection search to satisfy the power constraint $\sum_{j \in \mathcal{U}_{i}} \sum_{l=1}^{L_{j}}\left\|\mathbf{m}_{i, j, l}\right\|^{2} \leq P_{i}^{D}$. Similarly, the UL user transmit precoder $\mathbf{v}_{q, r, s}$ can be obtained as

$$
\mathbf{v}_{q, r, s}=\left(\boldsymbol{\Phi}_{r}+\lambda_{r} \mathbf{I}\right)^{-1} \mathbf{c}_{q, r, s}^{\mathrm{H}},
$$

where $\mathbf{c}_{q, r, s}=\alpha_{r} \beta_{r, s}^{U} \mathbf{w}_{r, s}^{\mathrm{H}} \mathbf{H}_{q, r}^{\mathrm{T}}, \lambda_{r}$ is the dual variable corresponding to the power constraint $\sum_{s=1}^{L_{r}}\left\|\mathbf{v}_{q, r, s}\right\|^{2} \leq P_{r}^{U}$, and $\boldsymbol{\Phi}_{r}$ is the UL user specific matrix given by

$$
\begin{aligned}
\boldsymbol{\Phi}_{r}= & \sum_{q \in \mathcal{B}_{U}} \sum_{r \in \mathcal{U}_{q}} \sum_{s=1}^{L_{r}} \alpha_{r} \beta_{r, s}^{U}\left(\mathbf{w}_{r, s}^{\mathrm{H}} \mathbf{H}_{q, r}^{\mathrm{T}}\right)^{\mathrm{H}} \mathbf{w}_{r, s}^{\mathrm{H}} \mathbf{H}_{q, r}^{\mathrm{T}} \\
& +\sum_{i \in \mathcal{B}_{D}} \sum_{j \in \mathcal{U}_{i}} \sum_{l=1}^{L_{j}} \alpha_{j} \beta_{j, l}^{D}\left(\mathbf{u}_{j, l}^{\mathrm{H}} \tilde{\mathbf{H}}_{r, j}\right)^{\mathrm{H}} \mathbf{u}_{j, l}^{\mathrm{H}} \tilde{\mathbf{H}}_{r, j} .
\end{aligned}
$$

The DL/UL precoder/decoder calculation steps are repeated until the convergence.

\section{TRAining And Signaling}

In the above beamformer design, the intermediate precoders/decoders and MSE user weights are needed to be

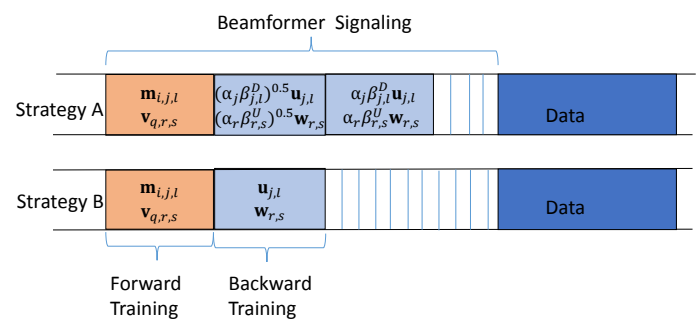

Fig. 1: TDD frame structure.

exchanged iteratively between the coordinated nodes. Hence, we employ OTA signaling for exchange this information [12], [14], [15]. However, in a dense, dynamic TDD network, a large number of orthogonal pilots required for exchanging the beamformer details perfectly. This is not possible with the limitations of the orthogonal resources. Therefore, we investigate the OTA signaling architecture under imperfect conditions by assuming that pilot training sequence used by each user are not orthogonal, and only their own pilot training sequence is known to each user.

In the OTA signaling scheme, we employ precoded pilots in both forward and backward directions. Here, the forward direction is referring when the DL BS/UL users are transmitting their training sequences, and the backward direction is referring when DL users/UL BSs are sending their training sequences. Furthermore, bi-directional signaling is embedded into each TDD frame to facilitate fast iterative information exchange [15]. Each coordinated node estimates their precoder/decoder based on the received precoded forward/backward pilots. Then, the estimated precoder/decoder is used for precoding the next forward/backward pilots. As we employ non-orthogonal and overlapping pilots, received effective channels are contaminated with each other. Also, each node is aware of their own pilot sequence only. Therefore, effective channels can not distinguish from the received information. Without this information, we can not construct the $\boldsymbol{\Phi}_{i}$ in (15) at the DL BS $i$ or $\boldsymbol{\Phi}_{r}$ in (18) at the UL user $r$ in order to estimate their transmit precoders. Hence, to overcome this problem, we introduce two different strategies to facilitate direct precoder/decoder estimation by using received precoded training information directly without decoding individual pilots. TDD frame structure for each strategy shown in Fig. 1.

\section{A. Strategy A}

In this strategy, we employ three training signals for one beamformer iteration, one in the forward direction and two consecutive training iterations in the backward direction. The forward training pilots are precoded with the transmit precoders $\mathbf{m}_{i, j, l} / \mathbf{v}_{q, r, s}$ at DL BS/UL users. Then, DL users/UL BSs estimate their MMSE receivers $\mathbf{u}_{j, l} / \mathbf{w}_{r, s}$ and corresponding user weights $\beta_{j, l}^{D} / \beta_{r, s}^{U}$ from the received information. Next, the first backward training signals are precoded with $\sqrt{\alpha_{j} \beta_{j, l}^{D}} \mathbf{u}_{j, l} / \sqrt{\alpha_{r} \beta_{r, s}^{U}} \mathbf{w}_{r, s}$ and the second backward training pilots are precoded with $\alpha_{j} \beta_{j, l}^{D} \mathbf{u}_{j, l} / \alpha_{r} \beta_{r, s}^{U} \mathbf{w}_{r, s}$. Both the 
backward pilot information is used to estimate the transmit precoder $\mathbf{m}_{i, j, l} / \mathbf{v}_{q, r, s}$ at DL BS/UL users. Detailed direct beamformer estimation procedure is described below.

\section{Transmit Precoder Estimation}

Let $\mathbf{b}_{j, l} \in \mathbb{C}^{S}$ denote the pilot training sequence for $l^{\text {th }}$ data stream of DL user $j$, where $S$ is the length of the pilot sequence. In the first backward training, pilots are precoded with $\sqrt{\alpha_{j} \beta_{j, l}^{D}} \mathbf{u}_{j, l} / \sqrt{\alpha_{r} \beta_{r, s}^{U}} \mathbf{w}_{r, s}$. The received precoded pilot training matrix at DL BS $i$ is given by

$$
\begin{aligned}
\mathbf{R}_{i}^{b_{1}}= & \sum_{b \in \mathcal{B}_{D}} \sum_{j \in \mathcal{U}_{b}} \sum_{l=1}^{L_{j}} \sqrt{\alpha_{j} \beta_{j, l}^{D}} \mathbf{H}_{i, j}^{\mathrm{H}} \mathbf{u}_{j, l} \mathbf{b}_{j, l}^{\mathrm{H}} \\
& +\sum_{q \in \mathcal{B}_{U}} \sum_{r \in \mathcal{U}_{q}} \sum_{s=1}^{L_{r}} \sqrt{\alpha_{r} \beta_{r, s}^{U}} \hat{\mathbf{H}}_{i, q}^{\mathrm{H}} \mathbf{w}_{r, s} \mathbf{b}_{r, s}^{\mathrm{H}}+\mathbf{N}_{i},
\end{aligned}
$$

where $\mathbf{N}_{i} \in \mathbb{C}^{M_{i} \times S}$ is the estimation noise matrix for all pilot symbols. In the second backward training, pilots are precoded with the weighted receivers $\alpha_{j} \beta_{j, l}^{D} \mathbf{u}_{j, l} / \alpha_{r} \beta_{r, s}^{U} \mathbf{w}_{r, s}$. The received pilot training matrix at the DL BS $i$ is given by

$$
\begin{aligned}
\mathbf{R}_{i}^{b_{2}}= & \sum_{b \in \mathcal{B}_{D}} \sum_{j \in \mathcal{U}_{b}} \sum_{l=1}^{L_{j}} \alpha_{j} \beta_{j, l}^{D} \mathbf{H}_{i, j}^{\mathrm{H}} \mathbf{u}_{j, l} \mathbf{b}_{j, l}^{\mathrm{H}} \\
& +\sum_{q \in \mathcal{B}_{U}} \sum_{r \in \mathcal{U}_{q}} \sum_{s=1}^{L_{r}} \alpha_{r} \beta_{r, s}^{U} \hat{\mathbf{H}}_{i, q}^{\mathrm{H}} \mathbf{w}_{r, s} \mathbf{b}_{r, s}^{\mathrm{H}}+\mathbf{N}_{i} .
\end{aligned}
$$

By using the received composite channel information $\mathbf{R}_{i}^{b_{1}}$, $\mathbf{R}_{i}^{b_{2}}$ and own pilot training sequence $b_{j, l}$, optimization problem at DL BS $i$ can be alternatively expressed as,

$$
\begin{array}{ll}
\min _{\mathbf{m}_{i, j, l}} & \sum_{j \in \mathcal{U}_{i} l=1} \sum_{i, j, l}^{L_{j}}\left(\mathbf{m}_{i}^{\mathrm{H}} \mathbf{R}_{i}^{b_{1}}\left(\mathbf{R}_{i}^{b_{1}}\right)^{\mathrm{H}} \mathbf{m}_{i, j, l}-2 \Re\left(\mathbf{m}_{i, j, l}^{\mathrm{H}} \mathbf{R}_{i}^{b_{2}} \mathbf{b}_{j, l}\right)\right) \\
\text { s. t. } & \sum_{j \in \mathcal{U}_{i}} \sum_{l=1}^{L_{j}}\left\|\mathbf{m}_{i, j, l}\right\|^{2} \leq P_{i} .
\end{array}
$$

This can be expanded using (19) and (20) as,

$$
\begin{aligned}
\min _{\mathbf{m}_{i, j, l}} & \sum_{j \in \mathcal{U}_{i}} \sum_{l=1}^{L_{j}}\left(-2 \Re\left(\alpha_{j} \beta_{j, l}^{D} \mathbf{u}_{j, l}^{\mathrm{H}} \mathbf{H}_{i, j} \mathbf{m}_{i, j, l}\right)+\psi_{i, j, l}\right. \\
& +\mathbf{m}_{i, j, l}^{\mathrm{H}}\left(\sum_{x \in \mathcal{B}_{D}} \sum_{y \in \mathcal{U}_{x}} \sum_{z=1}^{L_{y}} \alpha_{y} \beta_{y, z}^{D} \mathbf{H}_{x, y}^{\mathrm{H}} \mathbf{u}_{y, z}\left(\mathbf{H}_{x, y}^{\mathrm{H}} \mathbf{u}_{y, z}\right)^{\mathrm{H}}\right. \\
& \left.\left.+\sum_{q \in \mathcal{B}_{U}} \sum_{r \in \mathcal{U}_{q}} \sum_{s=1}^{L_{r}} \alpha_{r} \beta_{r, s}^{U} \hat{\mathbf{H}}_{i, q}^{\mathrm{H}} \mathbf{w}_{r, s}\left(\hat{\mathbf{H}}_{i, q}^{\mathrm{H}} \mathbf{w}_{r, s}\right)^{\mathrm{H}}\right) \mathbf{m}_{i, j, l}\right) \\
& \sum_{j \in \mathcal{U}_{i}} \sum_{l=1}^{L_{j}}\left\|\mathbf{m}_{i, j, l}\right\|^{2} \leq P_{i},
\end{aligned}
$$

where $\psi_{i, j, l}$ indicates the cross interference arrises from the pilots. This $\psi_{i, j, l}$ will be zero if orthogonal pilot sequences are used at each user. We can observe that the optimization problem in (14) and (22) are quite the same except the pilots cross interference term $\psi_{i, j, l}{ }^{1}$. Hence, with the knowledge of the received training matrices $\mathbf{R}_{i}^{b_{1}}, \mathbf{R}_{i}^{b_{2}}$ and own training

\footnotetext{
${ }^{1}$ The solutions are equivalent when the pilot sequences are orthogonal and the noise terms vanish.
}

sequences $\mathbf{b}_{j, l}$ we can estimate transmit beamformers in a closed form expressions as

$$
\mathbf{m}_{i, j, l}=\left(\mathbf{R}_{i}^{b_{1}}\left(\mathbf{R}_{i}^{b_{1}}\right)^{\mathrm{H}}+\mathbf{I} \nu_{i}\right)^{-1} \mathbf{R}_{i}^{b_{2}} \mathbf{b}_{j, l}
$$

where the optimal $\nu_{i}$ is found by bisection search to satisfy the power constraint $\sum_{j \in \mathcal{U}_{i}} \sum_{l=1}^{L_{j}}\left\|\mathbf{m}_{i, j, l}\right\|^{2} \leq P_{i}^{D}$.

Similarly, the received precoded pilot training matrix at UL user $r$ from the first backward training is given by,

$$
\begin{aligned}
\mathbf{R}_{r}^{b_{1}}= & \sum_{q \in \mathcal{B}_{U}} \sum_{t \in \mathcal{U}_{q}} \sum_{s=1}^{L_{t}} \sqrt{\alpha_{t} \beta_{t, s}^{U}}\left(\mathbf{w}_{t, s}^{\mathrm{H}} \mathbf{H}_{q, r}^{\mathrm{T}}\right)^{\mathrm{H}} \mathbf{b}_{t, s}^{\mathrm{H}} \\
& +\sum_{i \in \mathcal{B}_{D}} \sum_{j \in \mathcal{U}_{i}} \sum_{l=1}^{L_{j}} \sqrt{\alpha_{j} \beta_{j, l}^{D}}\left(\mathbf{u}_{j, l}^{\mathrm{H}} \tilde{\mathbf{H}}_{r, j}\right)^{\mathrm{H}} \mathbf{b}_{j, l}^{\mathrm{H}}+\mathbf{N}_{l},
\end{aligned}
$$

where $\mathbf{N}_{l} \in \mathbb{C}^{N_{r} \times S}$ is the estimation noise matrix for all pilot symbols. The received precoded training matrix in the second backward training is given by,

$$
\begin{aligned}
\mathbf{R}_{r}^{b_{2}}= & \sum_{q \in \mathcal{B}_{U}} \sum_{t \in \mathcal{U}_{q}} \sum_{s=1}^{L_{t}} \alpha_{t} \beta_{t, s}^{U}\left(\mathbf{w}_{t, s}^{\mathrm{H}} \mathbf{H}_{q, r}^{\mathrm{T}}\right)^{\mathrm{H}} \mathbf{b}_{t, s}^{\mathrm{H}} \\
& +\sum_{i \in \mathcal{B}_{D}} \sum_{j \in \mathcal{U}_{i}} \sum_{l=1}^{L_{j}} \alpha_{j} \beta_{j, l}^{D}\left(\mathbf{u}_{j, l}^{\mathrm{H}} \tilde{\mathbf{H}}_{r, j}\right)^{\mathrm{H}} \mathbf{b}_{j, l}^{\mathrm{H}}+\mathbf{N}_{l} .
\end{aligned}
$$

Then, by using the received training matrices $\mathbf{R}_{r}^{b_{1}}, \mathbf{R}_{r}^{b_{2}}$ and own training sequences $\mathbf{b}_{r, s}$, transmit precoders at UL user $r$ can be estimate directly as,

$$
\mathbf{v}_{q, r, s}=\left(\mathbf{R}_{r}^{b_{1}}\left(\mathbf{R}_{r}^{b_{1}}\right)^{\mathrm{H}}+\mathbf{I} \nu_{r}\right)^{-1} \mathbf{R}_{r}^{b_{2}} \mathbf{b}_{r, s}
$$

by bisection search over $\nu_{r}$ in such a way that the transmit power constraints $\sum_{s=1}^{L_{r}}\left\|\mathbf{v}_{q, r, s}\right\|^{2} \leq P_{q, r}^{U}$ hold.

\section{Receive beamformer and weights estimation}

In the forward training, pilots are precoded with the transmit precoders $\mathbf{m}_{i, j, l} / \mathbf{w}_{q, r, s}$. Then, the received precoded pilot training matrix at DL user $k$ is given by,

$$
\begin{aligned}
\mathbf{T}_{k}= & \sum_{i \in \mathcal{B}_{D}} \sum_{j \in \mathcal{U}_{i}} \sum_{l=1}^{L_{j}} \mathbf{H}_{i, k} \mathbf{m}_{i, j, l} \mathbf{b}_{j, l}^{\mathrm{H}} \\
& +\sum_{q \in \mathcal{B}_{U}} \sum_{r \in \mathcal{U}_{q}} \sum_{s=1}^{L_{r}} \tilde{\mathbf{H}}_{r, k} \mathbf{v}_{q, r, s} \mathbf{b}_{r, s}^{\mathrm{H}}+\mathbf{N}_{k}
\end{aligned}
$$

where $\mathbf{N}_{k} \in \mathbb{C}^{N_{k} \times S}$ is the estimation noise matrix for all pilot symbols. Similarly, the received precoded pilot training matrix at UL BS $p$ is given by

$$
\begin{aligned}
\mathbf{T}_{p}= & \sum_{q \in \mathcal{B}_{U}} \sum_{r \in \mathcal{U}_{q}} \sum_{s=1}^{L_{r}} \mathbf{H}_{p, r}^{\mathrm{T}} \mathbf{v}_{q, r, s} \mathbf{b}_{r, s}^{\mathrm{H}} \\
& +\sum_{i \in \mathcal{B}_{D}} \sum_{j \in \mathcal{U}_{i}} \sum_{l=1}^{L_{j}} \hat{\mathbf{H}}_{i, p} \mathbf{m}_{i, j, l} \mathbf{b}_{j, l}^{\mathrm{H}}+\mathbf{N}_{p} .
\end{aligned}
$$

Then, by using the received composite channel information $\mathbf{T}_{k} / \mathbf{T}_{p}$ and own pilot training sequence $\mathbf{b}_{j, l} / \mathbf{b}_{r, s}$ we can directly estimate the MMSE receivers, from the formula in (29) for the DL user $k$ and from the formula in (30) for the $\mathrm{UL}$ BS $p$ as

$$
\begin{aligned}
\mathbf{u}_{k, l} & =\left(\mathbf{T}_{k} \mathbf{T}_{k}^{\mathrm{H}}\right)^{-1} \mathbf{T}_{k} \mathbf{b}_{k, l}, \\
\mathbf{w}_{r, s} & =\left(\mathbf{T}_{p} \mathbf{T}_{p}^{\mathrm{H}}\right)^{-1} \mathbf{T}_{p} \mathbf{b}_{r, s},
\end{aligned}
$$




$$
\begin{aligned}
& f\left(\mathbf{m}_{i, j, l}\right)=\sum_{j \in \mathcal{U}_{i}} \sum_{l=1}^{L_{j}}\left(\mathbf{m}_{i, j, l}^{\mathrm{H}}\left(\mathbf{R}_{i}^{b_{1}}\left(\mathbf{I}+\sum_{u \in \mathcal{U}_{i}} \sum_{v=1}^{L_{u}}\left(\frac{\alpha_{u} \beta_{u, v}^{D}-1}{S}\right) \mathbf{b}_{u, v} \mathbf{b}_{u, v}^{\mathrm{H}}\right)\left(\mathbf{R}_{i}^{b_{1}}\right)^{\mathrm{H}}\right) \mathbf{m}_{i, j, l}-2 \Re\left(\alpha_{j} \beta_{j, l}^{D} \mathbf{m}_{i, j, l}^{\mathrm{H}} \mathbf{R}_{i}^{b_{1}} \mathbf{b}_{j, l}\right)\right) \\
& f\left(\mathbf{m}_{i, j, l}\right)= \sum_{j \in \mathcal{U}_{i}} \sum_{l=1}^{L_{j}}\left(-2 \Re\left(\alpha_{j} \beta_{j, l}^{D} \mathbf{u}_{j, l}^{\mathrm{H}} \mathbf{H}_{i, j} \mathbf{m}_{i, j, l}\right)+\psi_{i, j, l}+\mathbf{m}_{i, j, l}^{\mathrm{H}}\left(\sum_{j \in \mathcal{U}_{i}} \sum_{l=1}^{L_{j}} \alpha_{j} \beta_{j, l}^{D} \mathbf{H}_{i, j}^{\mathrm{H}} \mathbf{u}_{j, l}\left(\mathbf{H}_{i, j}^{\mathrm{H}} \mathbf{u}_{j, l}\right)^{\mathrm{H}}\right.\right. \\
&\left.\left.+\sum_{x \in \mathcal{B}_{D} /\{i\}} \sum_{y \in \mathcal{U}_{x}} \sum_{z=1}^{L_{y}} \mathbf{H}_{x, y}^{\mathrm{H}} \mathbf{u}_{y, z}\left(\mathbf{H}_{x, y}^{\mathrm{H}} \mathbf{u}_{y, z}\right)^{\mathrm{H}}+\sum_{q \in \mathcal{B}_{U}} \sum_{r \in \mathcal{U}_{q}} \sum_{s=1}^{L_{r}} \hat{\mathbf{H}}_{i, q}^{\mathrm{H}} \mathbf{w}_{r, s}\left(\hat{\mathbf{H}}_{i, q}^{\mathrm{H}} \mathbf{w}_{r, s}\right)^{\mathrm{H}}\right) \mathbf{m}_{i, j, l}\right)
\end{aligned}
$$

respectively. Also, MSE values for the DL user and UL BS can be estimated using (31) and (32), respectively, as

$$
\begin{gathered}
\tilde{\epsilon}_{k, l}^{D}=1-\left(\mathbf{u}_{k, l}\right)^{\mathrm{H}} \mathbf{T}_{k} \mathbf{b}_{k, l}, \\
\tilde{\epsilon}_{r, s}^{U}=1-\left(\mathbf{w}_{r, s}\right)^{\mathrm{H}} \mathbf{T}_{p} \mathbf{b}_{r, s} .
\end{gathered}
$$

Note that here we assume that for a particular user forward and backward sequences are same. However, the forward/backward pilots can be designed separately.

\section{B. Strategy $B$}

In contrast to Strategy A, this method needs only one backward training iteration for one beamformer iteration. In practice, it is more convenient to have two training iterations instead of three. Similar to Strategy A, forward training pilots are precoded with the transmit precoders $\mathbf{m}_{i, j, l} / \mathbf{v}_{q, r, s}$ at DL BS/UL users. Then, DL users/UL BSs estimate the MMSE receivers beamformers $\mathbf{u}_{j, l} / \mathbf{w}_{r, s}$. Next, the estimated MMSE receivers $\mathbf{u}_{j, l} / \mathbf{w}_{r, s}$ are used to the precode backward training pilots. The information received from the backward training pilots are not enough to reconstruct the $\boldsymbol{\Phi}_{i} / \boldsymbol{\Phi}_{r}$ in (15) and (18). However, we approximately determine the transmit precoders by using the locally estimated MSE weights.

\section{Transmit Precoder Estimation}

Here, the backward training sequence is precoded with the MMSE receivers $\mathbf{u}_{j, l} / \mathbf{w}_{r, s}$. Hence, the received precoded pilot training matrix at BS $i$ is given by

$$
\begin{aligned}
\mathbf{R}_{i}^{b_{1}}= & \sum_{b \in \mathcal{B}_{D}} \sum_{j \in \mathcal{U}_{b}} \sum_{l=1}^{L_{j}} \mathbf{H}_{i, j}^{\mathrm{H}} \mathbf{u}_{j, l} \mathbf{b}_{j, l}^{\mathrm{H}} \\
& +\sum_{q \in \mathcal{B}_{U}} \sum_{r \in \mathcal{U}_{q}} \sum_{s=1}^{L_{r}} \hat{\mathbf{H}}_{i, q}^{\mathrm{H}} \mathbf{w}_{r, s} \mathbf{b}_{r, s}^{\mathrm{H}}+\mathbf{N}_{i} .
\end{aligned}
$$

Furthermore, we assume $\alpha_{j}$ is locally available and $\beta_{j, l}^{D}$ is locally estimated. We can estimate these local weights $\beta_{j, l}^{D}$ refering to (13) as

$$
\beta_{j, l}^{D}=1 /\left(1-\left(\mathbf{R}_{i}^{b_{1}} \mathbf{b}_{j, l}\right)^{\mathrm{H}} \hat{\mathbf{m}}_{i, j, l}\right)
$$

where $\hat{\mathbf{m}}_{i, j, l}$ is the transmit precoder estimated in the previous iteration or the initial condition. In order to estimate transmit precoders $\mathbf{m}_{i, j, l}$ at DL BS $i$, we construct objective function $f\left(\mathbf{m}_{i, j, l}\right)$ by using received pilot matrix $\mathbf{R}_{i}^{b_{1}}$ and training sequence $\mathbf{b}_{j, l}$ as in (35) top of the page. Then (35) can be expanded into (36) by substituting (33) as in top of the page. There, $\psi_{i, j, l}$ indicates the cross interference arises from pilots. It is clear that objectives in (36) and (14) are not equal. This is mainly due to unavailability of the priority weights and MSE weights from the interfering users. However, we can obtaine a closed form solution for $\mathbf{m}_{i, j, l}$ from the approximated objective in (35) as,

$$
\begin{aligned}
\mathbf{m}_{i, j, l}= & \left(\mathbf{R}_{i}^{b_{1}}\left(\mathbf{I}+\sum_{u \in \mathcal{U}_{i}} \sum_{v=1}^{L_{u}}\left(\frac{\alpha_{u} \beta_{u, v}^{D}-1}{S}\right) \mathbf{b}_{u, v} \mathbf{b}_{u, v}^{\mathrm{H}}\right)\left(\mathbf{R}_{i}^{b_{1}}\right)^{\mathrm{H}}\right. \\
& \left.+\mathbf{I} \nu_{i}\right)^{-1} \alpha_{j} \beta_{j, l}^{D} \mathbf{R}_{i}^{b_{1}} \mathbf{b}_{j, l}
\end{aligned}
$$

where $\nu_{i}$ is found by bisection search to satisfy the power constraint $\sum_{j \in \mathcal{U}_{i}} \sum_{l=1}^{L_{j}}\left\|\mathbf{m}_{i, j, l}\right\|^{2} \leq P_{i}^{D}$.

A similar result can be obtained for UL users as well. However, the derivations are omitted due to space limitation. The receive beamformer estimation procedure for Strategy B is same as the approach used in Strategy A.

\section{Numerical EXAMPLES}

In the simulation model, we consider a sectore seven cell wrap-around model with three sectors (21 BSs) and two users per sector. The distance between adjacent BSs is $200 \mathrm{~m}$, and the path loss exponent is 3.67. The number of antennas at each BS is 4 , and the number of antennas at each user terminal is 2. To simplifying the analysis, all the user priority weights are assumed to be 1 and, the maximum number of the spatial stream is 1 . All the simulations are averaged over 1000 i.i.d channel realizations. Transmit power is setup such a way that the signal-to-noise ratio (SNR) of UL/DL cell edge user is $12 \mathrm{~dB} / 15 \mathrm{~dB}$. Furthermore, pilot gain considered to be $10 \mathrm{~dB}$ during the channel estimation.

We consider two pilot allocation schemes. The first generates non-orthogonal random pilot sequences for the given length. However, same cell users are always assigned with orthogonal sequences. We refer this scheme as Random Pilots Assignment (RPA). The second scheme generates orthogonal pilot sequences for the considered sequence length. For the sequence lengths $8,16, \ldots, 64$, we can create the matching number of orthogonal sequences. Then, these orthogonally generated pilots are randomly re-used among users. We refer this scheme as Pilot Reuse (PR). Also, the proposed direct estimation DE algorithm is compared with stream specific estimation (SSE). In SSE method, we decode all the pilots separately to (user should know all the pilot training sequences used at other nodes) reconstruct (22), (4), (36) and (8) (user should know all the pilot training sequences used at other nodes) [12].

Fig. 2 compares the sum rate of the both Strategy A and B with the different pilot allocations. For both pilot allocation schemes, Strategy B performs much better in the shorter 


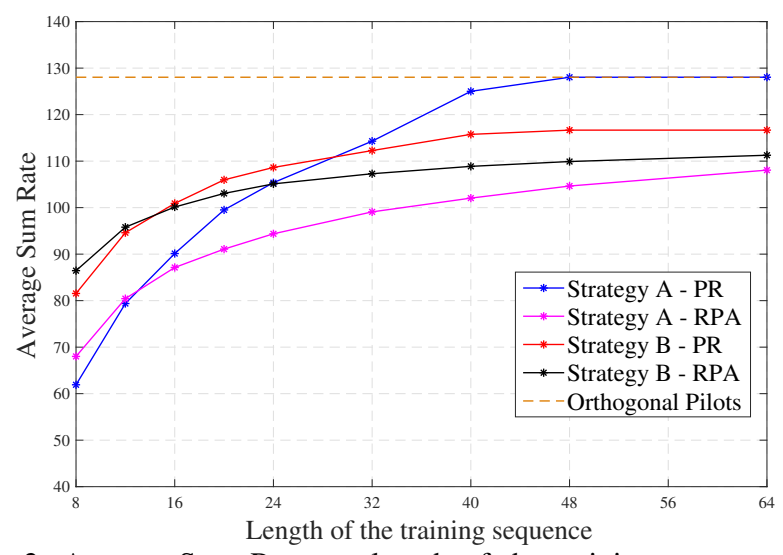

Fig. 2: Average Sum Rate vs. length of the training sequence for different pilot allocations with 30 DL users and 12 UL users.

sequence length in comparison to Strategy A. With short pilots, the MSE weight and precoder estimation error aggregate in the precoded pilots of Strategy A, which causes some performance deterioration. Locally estimated MSE weights and a single set of pilots per iteration makes Strategy B more robust to severely limited pilot sequence lengths. However, the performance of the Strategy A improves with the sequence length. Strategy A has considerable performance gain over Strategy B for the PR case when sequence length is larger than 28 . This is mainly due to inability to recover the user MSE weights from the interfering users. Fig. 3 illustrates the performance of each strategy with the DE and SSE for the sequences obtained from RPA. Most of the sequence lengths, DE-based design performs better than the SSE-based design. This is due to the additive estimation noise in the decoding and reconstructing process in the SSE.

\section{CONCLUSIONS}

In this paper, WSR maximization is considered for dynamic TDD systems with user-specific and BS specific power constraints. The original NP-hard problem was solved iteratively in a decentralized manner. Bi-directional signaling strategies were applied to facilitate efficient OTA signaling. The proposed strategies utilized direct beamformer estimation to allow non-orthogonal pilot allocation and ability to withstand pilot contamination. Numerical examples investigate the performance of each strategy for different pilot sequence lengths versus random pilot allocation. Strategy $B$ was shown to performs much better for shorter sequence length. On the other hand, Strategy A provided better performance with longer pilot sequences.

\section{REFERENCES}

[1] T. Nakamura, S. Nagata, A. Benjebbour, Y. Kishiyama, T. Hai, S. Xiaodong, Y. Ning, and L. Nan, "Trends in small cell enhancements in LTE advanced," IEEE Commun. Mag., vol. 51, no. 2, pp. 98-105, Feb. 2013.

[2] J. Li, S. Farahvash, M. Kavehrad, and R. Valenzuela, "Dynamic TDD and fixed cellular networks," IEEE Commun. Lett., vol. 4, no. 7, pp. 218-220, Jul. 2000.

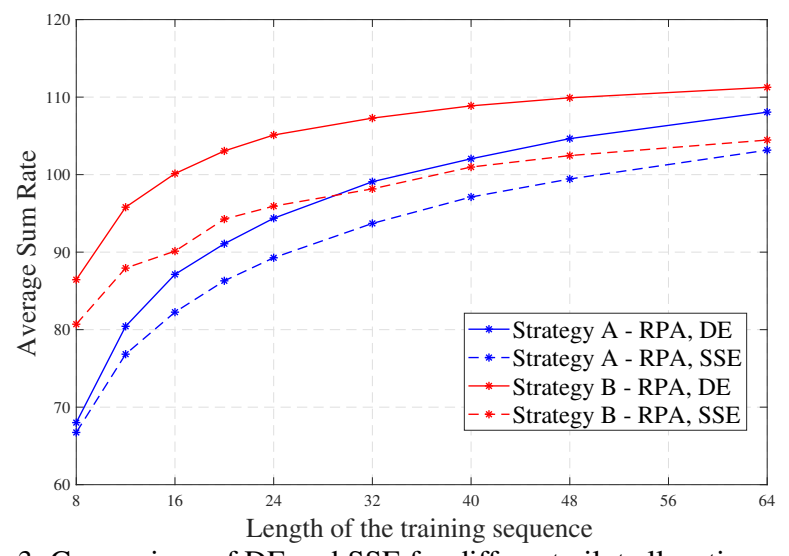

Fig. 3: Comparison of DE and SSE for different pilot allocations with 30 DL users and 12 UL users.

[3] Z. Shen, A. Khoryaev, E. Eriksson, and X. Pan, "Dynamic uplinkdownlink configuration and interference management in TD-LTE," IEEE Commun. Mag., vol. 50, no. 11, pp. 51-59, Nov. 2012.

[4] B. Yu, S. Mukherjee, H. Ishii, and L. Yang, "Dynamic TDD support in the LTE-B enhanced Local Area architecture," in IEEE Globecom Workshops (GC Wkshps), 2012, pp. 585-591.

[5] 3GPP TSG RAN WG1, "Study on scenarios and requirements for next generation access technologies TR 38.913," Tech. Rep., 3rd Generation Partnership Project 3GPP, www.3gpp.org, 2016.

[6] M. S. ElBamby, M. Bennis, W. Saad, and M. Latva-aho, "Dynamic Uplink-Downlink Optimization in TDD-based Small Cell Networks," in IEEE 11th International Symposium on Wireless Communication Systems (ISWCS), 2014.

[7] P. Omiyi, H. Haas, and G. Auer, "Analysis of TDD cellular interference mitigation using busy-bursts," IEEE Trans. Wireless Commun., vol. 6 , no. 7, pp. 2721-2731, Jul. 2007.

[8] I. Sohn, K. B. Lee, and Y. Choi, "Comparison of decentralized time slot allocation strategies for asymmetric traffic in TDD systems," IEEE Trans. Wireless Commun., vol. 8, no. 6, pp. 2990-3003, Jun. 2009.

[9] H. Chung, M. Kim, N. Kim, and S. Yun, "Time slot allocation based on region and time partitioning for dynamic TDD-OFDM systems," in IEEE 63rd Vehicular Technology Conference (VTC), 2006, vol. 5, pp. 2459-2463.

[10] S. S. Christensen, R. Agarwal, E. Carvalho, and J. M. Cioffi, "Weighted sum-rate maximization using weighted MMSE for MIMO-BC beamforming design," IEEE Trans. Wireless Commun., vol. 7, no. 12, pp. 4792-4799, Dec. 2008.

[11] Q. Shi, M. Razaviyayn, Z. Luo, and C. He, "An iteratively weighted MMSE approach to distributed sum-utility maximization for a MIMO interfering broadcast channel," IEEE Trans. Signal Processing, vol. 59, no. 9, pp. 4331-4340, Sept. 2011.

[12] P. Komulainen, A. Tölli, and M. Juntti, "Effective CSI signaling and decentralized beam coordination in TDD multi-cell MIMO systems," IEEE Trans. Signal Processing, vol. 61, no. 9, pp. 2204-2218, May. 2013.

[13] G. Venkatraman, A. Tölli, M. Juntti, and L. N. Tran, "Traffic aware resource allocation schemes for multi-cell MIMO-OFDM systems," IEEE Trans. Signal Processing, vol. 64, no. 11, pp. 2730-2745, Jun. 2016.

[14] P. Jayasinghe, A. Tölli, J. Kaleva, and M. Latva-aho, "Bi-directional signaling for dynamic TDD with decentralized beamforming," in IEEE Int. Conf. Commun. Workshop (ICCW), 2015, pp. 185-190.

[15] P. Jayasinghe, A. Tölli, and M. Latva-aho, "Bi-directional signaling strategies for dynamic TDD networks," in IEEE 16th Int. Wkshp Sig. Proc. Adv. Wireless Commun. (SPAWC), 2015, pp. 540-544.

[16] E. G. Larsson, O. Edfors, F. Tufvesson, and T. L. Marzetta, "Massive mimo for next generation wireless systems," IEEE Commun. Mag., vol. 52, no. 2, pp. 186-195, Feb. 2014

[17] C. Shi, R. A. Berry, and M. L. Honig, "Bi-Directional Training for Adaptive Beamforming and Power Control in Interference Networks," IEEE Trans. Signal Processing, vol. 62, pp. 607-618, Feb. 2014. 